\title{
Management of Aggressive Periodontitis by Interdisciplinary Approach: A Case Report
}

\author{
Manab Kosala, Vishal Siwach, A. K. Jha, Navneet Sheokand \\ Department of Dental Surgery, Armed Forces Medical College, Sholapur Road, Pune, Maharashtra, India \\ E-mail for correspondences: Navneet_y2n@yahoo.com
}

\begin{abstract}
The primary objective of periodontal therapy is to restore and maintain the health and integrity of the attachment apparatus of teeth. In adults, the loss of teeth or periodontal support can result in pathological teeth migration involving either a single tooth or a group of teeth. This may result in the development of a median diastema or general spacing of the teeth with or without incisal proclination, rotation or tipping of bicuspids and molars with the collapse of the posterior occlusion, and decreasing vertical dimension. Adjunctive orthodontic therapy is necessary to resolve these problems. Alignment of crowded or malposed teeth should be an advantage to clean up all surfaces of the teeth, which is essential care for the periodontitis. The present case report discusses the clinical presentation and treatment carried out in a case of generalized aggressive periodontitis with pathological migration of teeth by combined periodontal and orthodontic therapy.
\end{abstract}

Key words: Aggressive periodontitis, orthodontics, periodontics, tooth movement.

\section{INTRODUCTION}

The primary objective of periodontal therapy is to restore and maintain the health and integrity of the attachment apparatus of teeth. In adults, the loss of teeth or periodontal support can result in pathological teeth migration involving either a single tooth or a group of teeth. This may result in the development of a median diastema or general spacing of the teeth with or without incisal proclination, rotation or tipping of bicuspids and molars with the collapse of the posterior occlusion, and decreasing vertical dimension. Adjunctive orthodontic therapy is necessary to resolve these problems. ${ }^{[1]}$

The present case report discusses the clinical presentation and treatment carried out in a case of generalized aggressive periodontitis by combined periodontal and orthodontic therapy.

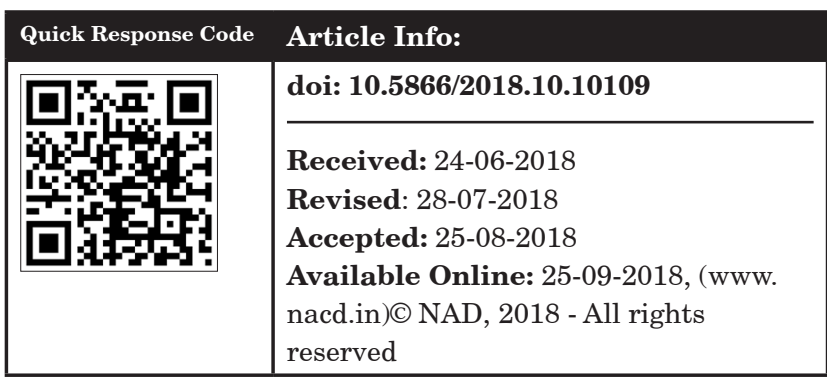

\section{CASE REPORT}

A 34-year-old female patient presented with chief complaint of unesthetic appearance due to forwardly placed upper front teeth with increased spacing for 2-3 years [Figure 1]. The patient added that mobility and pus discharge from upper front teeth were gradually increasing. Patient's medical history was non-contributory. Extraoral examination revealed that face was bilaterally symmetrical and did not show any signs of systemic illness. The patient stated that her mother lost her teeth at an early age. Intraoral examination showed good oral hygiene. In the upper anterior region, gingiva was reddish in color, soft, and edematous in consistency with rolled-out margin and absence of stippling. Bleeding on probing was present. Pocket depth of $10 \mathrm{~mm}$ was measured in 13,21 , and 23 and $6-8 \mathrm{~mm}$ in 11,12 , and 22 regions. Pocket depth varied from $5 \mathrm{~mm}$ to $6 \mathrm{~mm}$ in maxillary and mandibular molars. Maxillary central incisors were showing Grade-II mobility, whereas maxillary lateral incisors and canines were showing Grade-I mobility. Maxillary anterior teeth showed signs of pathological migration with a recession of $2 \mathrm{~mm}$ in the region of 11 . Vitality test showed upper anterior teeth to be vital. Investigations were carried out to rule out systemic involvement. Orthopantomogram [Figure 2] and lateral cephalogram were done to assess the bony 
topography. Based on the history, clinical features, and radiological findings, the case was diagnosed as generalized aggressive periodontitis.

The patient was educated and motivated. Scaling and root planing were done and the case was evaluated for 4 weeks. Undisplaced flap surgery in region from 14 to 24 [Figure 3], 31 to 38 , and 41 to 48 was carried out and splinting was done from 13 to 23 region.

Post-operative recall and evaluation of oral hygiene were carried out for 6 months. The patient showed $3 \mathrm{~mm}$ gain in clinical attachment and reduction in inflammation. The study casts were made and the case was evaluated orthodontically for esthetic correction. The case was taken up for fixed orthodontic therapy. Very light orthodontic forces were used to bodily retract and intrude incisors, and various modifications were done in mechanotherapy to apply force closer to center of resistance of anterior segment including the use of palatal microimplant. During orthodontic therapy, regular scaling and

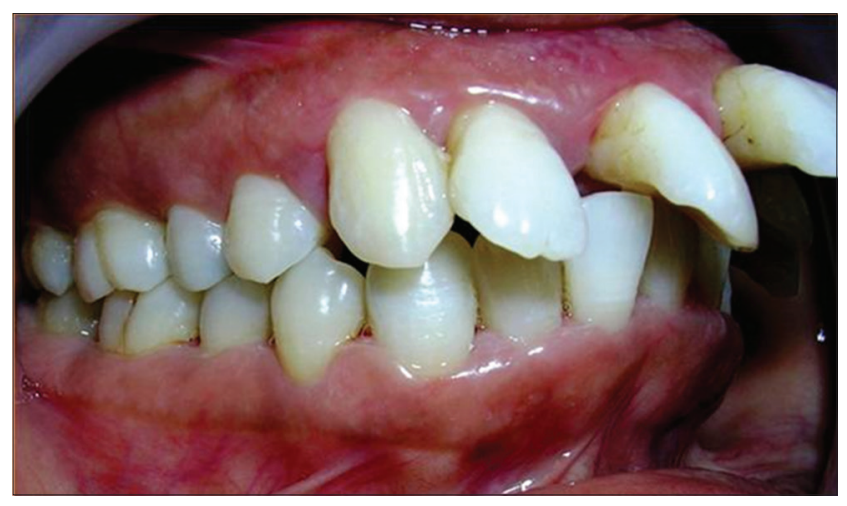

Figure 1: Pre-operative picture showing forwardly placed upper front teeth with increased spacing

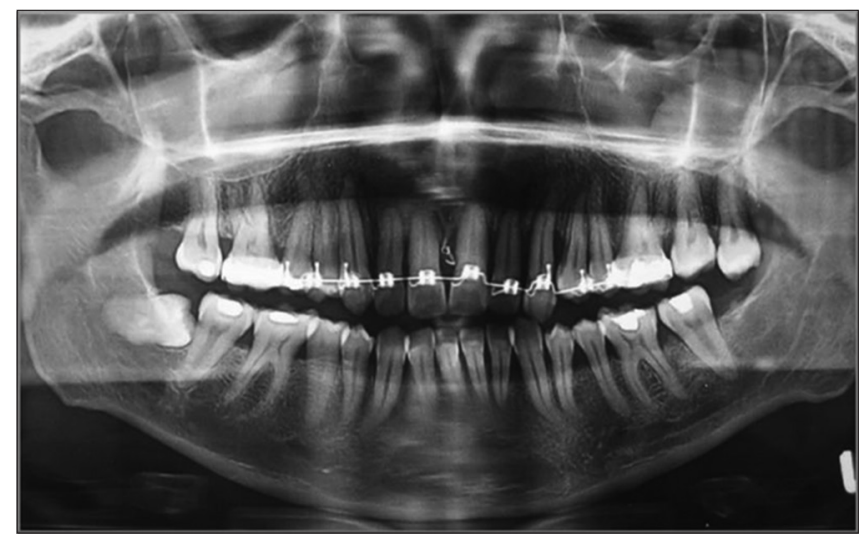

Figure 2: OPG showing bone loss due to aggressive periodontitis in the maxillary anterior teeth root planing were carried out and oral hygiene instructions were reinforced. The appliance was debonded after 8 months, and fixed palatal retention was given using fiber reinforced composite strips. At the end of therapy, the patient showed remarkable improvement in facial appearance, gain in clinical attachment level, and reduction in mobility of upper anterior teeth [Figure 4].

\section{DISCUSSION}

A common problem in adult patients with periodontal disease is the migration, elongation, and spacing of incisors. ${ }^{[2]}$ Periodontitis affected patients in younger age as shown in this case needs an improvement in the quality of life with esthetic and functional management.

Several reports have suggested that orthodontic force can have negative effects such as loss of attachment and further bone destruction when periodontal inflammation is

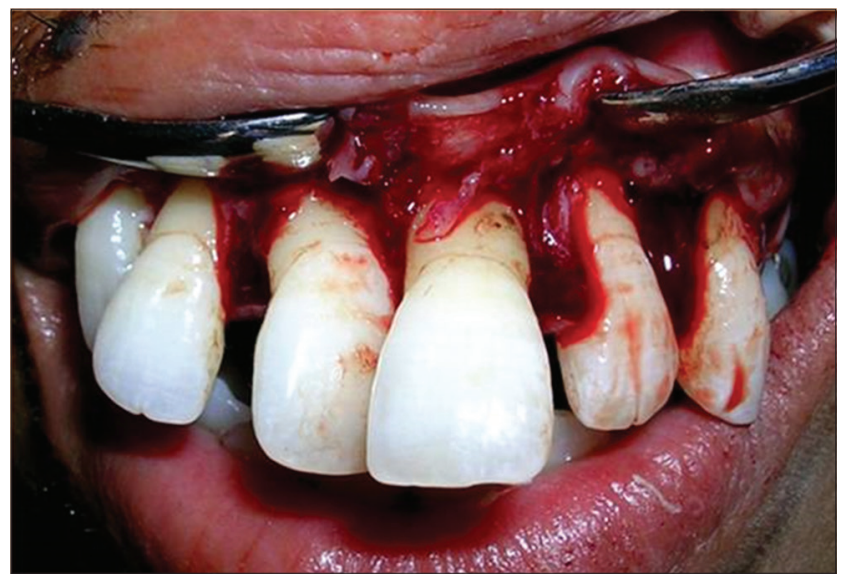

Figure 3: Undisplaced flap surgery was performed from 14 to 24

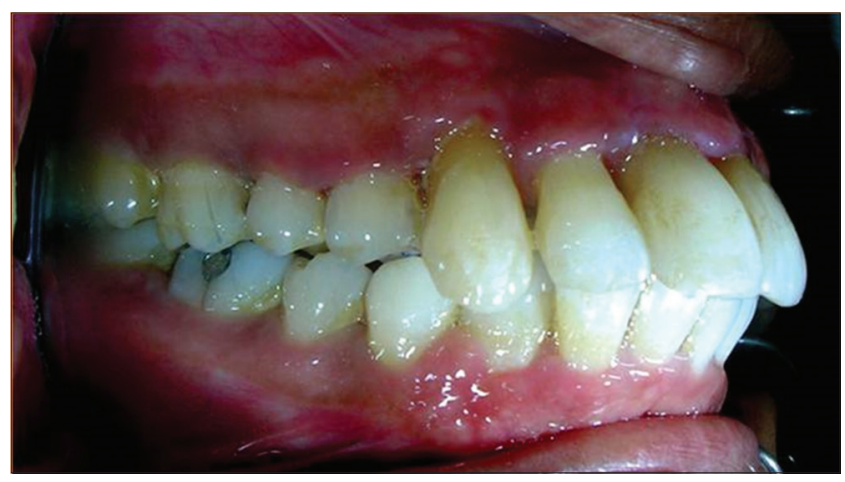

Figure 4: Post-operative picture showing remarkable improvement in facial appearance, gain in clinical attachment level, and reduction in mobility of upper anterior teeth 
present. ${ }^{[3]}$ An experimental study has also shown that regeneration of the periodontal ligament does not occur in the tissue with inflammation and that degenerated tissues produced in the pressure side of tooth movement are defenseless to the bacterial infection. ${ }^{[4,5]}$ Therefore, it is essential to eliminate inflammation of periodontal tissues before orthodontic treatment. In the present case, an intensive care was made to recover the periodontal environment with no inflammation before the orthodontic treatment, closely to its normal circumstance so that the tissue response could be the same as that in normal periodontal tissues free from infections.

To eliminate the source of the infection scaling, root planning and flap operation were performed before the orthodontic treatment along with elimination of subgingival calculus and plaque.

Special considerations must be given to force application, particularly its magnitude and duration, in a dentition that demonstrates a reduced periodontal support mechanism. ${ }^{[6]}$

If the patient has lost bone support, the center of resistance moves apically, and the same force against the crown produces greater pressure in the periodontal ligament of a periodontally compromised tooth than in a normally supported one intrusion of elongated incisors which is the logical solution to close the anterior diastema. ${ }^{[7,8]}$ Due to the risks of loss of the bone support around teeth and root resorption during intrusion, very light forces should be applied. ${ }^{[9]}$

Alignment of crowded or malposed teeth should be an advantage to clean up all surfaces of the teeth, which is essential care for the periodontitis. ${ }^{[10]}$ Orthodontic treatment also contributes to the promotion of oral health of the patient. As a result, healthy periodontal condition was maintained.

\section{CONCLUSION}

Periodontal health is essential for any form of dental treatment. Adult patients must undergo meticulous oral hygiene care and periodontal maintenance to maintain healthy gingival tissue during active orthodontic treatment. Adult orthodontic tooth movement can be performed on both healthy and diseased periodontium with few detrimental effects (root resorption), providing that physiologic forces are used. Interdisciplinary cooperation in both disciplines can work in the patient's best interest to transform unattractive dentitions showing spaced, extruded, or otherwise migrated teeth in inflamed and reduced periodontium into persons with attractive, esthetic dentitions, and smiles.

\section{REFERENCES}

1. Ong MA, Wang HL, Smith FN. Interrelationship between periodontics and adult orthodontics. J Clin Periodontol 1998;25:271-7.

2. Miller BH. Orthodontics for the adult patient. Part 1. - Introduction. Br Dent J 1980;148:97-100.

3. Wennström JL, Stokland BL, Nyman S, Thilander B. Periodontal tissue response to orthodontic movement of teeth with infrabony pockets. Am J Orthod Dentofacial Orthop 1993;103:313-9.

4. Ericsson I, Thilander B, Lindhe J, Okamoto H. The effect of orthodontic tilting movements on the periodontal tissues of infected and non-infected dentitions in dogs. J Clin Periodontol 1977;4:278-93.

5. Nakamura Y, Tanaka T, Noda K, Shimpo S, Oikawa T, Hirashita A, et al. Calcification of degenerating tissues in the periodontal ligament during tooth movement. J Periodontal Res 2003;38:343-50.

6. Polson A, Caton J, Polson AP, Nyman S, Novak J, Reed B, et al. Periodontal response after tooth movement into intrabony defects. J Periodontol 1984;55:197-202.

7. Burstone CJ, Pryputniewicz RJ. Holographic determination of centers of rotation produced by orthodontic forces. Am J Orthod 1980;77:396-409.

8. Proffit WR, Fields HW, editors. Special considerations in comprehensive treatment of adults. In: Contemporary Orthodontics. $2^{\text {nd }}$ ed. St. Louis: Mosby; 1993. p. 585-606.

9. Vanarsdall RL, Musich DR. Adult orthodontics: Diagnosis and treatment. In: Graber TM, Vanarsdall RL, editors. Orthodontics Current Principles and Techniques. $2^{\text {nd }}$ ed. St. Louis: Mosby; 1994. p. 750-834.

10. Re S, Corrente G, Abundo R, Cardaropoli D. Orthodontic treatment in periodontally compromised patients: 12 -year report. Int J Periodontics Restorative Dent 2000;20:31-9. 